\title{
OUTSOURCING AND SUPPLY CHAINS \\ CONCLUSIONS
}

\author{
Juan Peña Moncho \\ Trainee in Ibáñez Labor Lawyers \\ Andrés Camargo Rodríguez \\ PhD student, Universitat Pompeu Fabra
}

\begin{abstract}
The Comparative Labor Dossier (CLLD) in this issue 1/2019 of IUSLabor is dedicated to outsourcing and supply chains. Aside from Spain, renowned academics and professionals from the following countries have participated in this publication: France, Germany, Italy, Portugal, United Kingdom, Brazil, Costa Rica, Panamá, Peru, Uruguay, Canada, China and India. The following pages contain the 10 main conclusions reached in the comparative study. Nevertheless, it is highly recommended the detailed reading of the pertinent chapters to better understand the conclusions here indicated. Likewise, you will find attached to the conclusions a summary table with the answers of the different legal regimes to each one of the questions on outsourcing and supply chains analyzed in this issue of IUSLabor.

El Comparative Labor Law Dossier (CLLD) de este número 1/2019 de IUSLabor está dedicado la subcontratación y a las cadenas de producción. Además de España, en esta publicación han participado académicos y profesionales de reconocido prestigio de los siguientes países: Alemania, Francia, Italia, Portugal, Reino Unido, Brasil, Costa Rica, Panamá, Perú, Uruguay, Canadá, China e India. En las siguientes páginas hemos incluido las 10 conclusiones principales que hemos alcanzado. No obstante, recomendamos encarecidamente una lectura detallada de los capítulos correspondientes para una mejor comprensión de los puntos aquí señalados. Asimismo, las conclusiones vienen acompañadas de un cuadro-resumen con las respuestas de los distintos ordenamientos jurídicos a cada una de las preguntas sobre las consecuencias laborales de la subcontratación y las cadenas productivas analizadas en este número de IUSLabor.
\end{abstract}

Tittle: Outsourcing and supply chains - Conclussions

Título: Subcontratación y cadenas de producción. Conclusiones

IUSLabor 1/2019, ISSN 1699-2938, p. 149-186.

DOI. 10.31009/IUSLabor.2019.101.02 
Keyworkds: outsourcing, supply chains, joint liability, principle of equal treatment, Temporary Employment Agencies.

Palabras clave: subcontratación, cadenas de producción, responsabilidad solidaria, principio de igualdad de trato, Empresas de Trabajo Temporal

\section{Summary}

1. «Top ten» conclusions

2. «Top ten» conclusions

3. Summary table

3.1. Europe

3.2. Latin-America

3.3. North America

3.4. Asia 


\section{1. «Top ten» conclusions}

The Comparative Labor Law Dossier (CLLD) in this issue 1/2019 of IUSLabor is dedicated to outsourcing and supply chains and it includes articles elaborated by several international renowned academics and professionals regarding this important matter.

As all labour jurist know, externalisation of production and services has become a widespread practice in most of the countries and economic sectors. Therefore, the aim of this CLLD is to analyse, from a comparative perspective, how countries are approaching their labor regulation on this matter. Concretely, the countries analysed will be France, Germany, Italy, Portugal, Spain, United Kingdom, Brazil, Costa Rica, Dominican Republic, Panamá, Peru, Uruguay, Canada, China and India.

Specifically, academics have participated in this CLLD by answering the following questions:

1. Is it outsourcing a legal form of production organisation?

2. Are there limits and/or prohibitions to outsourcing?

3. Does the company that partly or totally outsources its production have any labor or Social Security responsibility towards the subcontractor's workers? What responsibilities?

4. What is the collective bargaining agreement applicable to the contractor during its relationship with the main company?

5. Is the subcontractor legally obliged to recognize its workers the same labour conditions applicable to the user company?

6. In which cases is outsourcing considered fraudulent or there is an illegal transfer of workers? What are the consequences?

7. Is the hiring of workers through Temporary Employment Agencies allowed in your country? If so, in which cases?

8. Are there specific cases or economic activities in which hiring workers through Temporary Employment Agencies is limited and/or prohibited?

9. What labor and Social Security liabilities do Temporary Employment Agencies have with respect to the workers hired and transferred to user firms? And the user firm?

10. How are the labor conditions applicable to workers hired by Temporary Employment Agencies and transferred to user companies determined?

Next, it shall be examined the $\mathbf{1 0}$ most important conclusions regarding these questions, following the same order presented above, in accordance with the articles written by our international consultants. 
1. In all of the European, American and Asian countries analysed outsourcing is a legal form of production organisation. Additionally, many of the countries analysed acknowledge this legal activity as an expression of entrepreneurial freedom protected by their constitutions.

It is worth highlighting the recent introduction of legislation in the Brazilian legal system, where productive decentralization went from being considered illegal by case law, to be accepted by the legal norms adopted in 2017 .

2. However, despite the fact that in all of these countries outsourcing is a legal activity, some countries introduce prohibitions and limitations to this form of externalisation. Nevertheless, each country has its own specificities.

The only European country that foresees prohibitions on outsourcing is Italy, where risk-taking activities are not allowed to be outsourced. Additionally, collective bargaining agreements are entitled to introduce more prohibitions or limitations. Other European Union countries establish limitations regarding the construction sector, such as France or Spain. Germany, for instance, has recently passed a law introducing limitations to outsourcing measures requiring the collection, processing or use of personal data. Conversely, neither Portugal nor the United Kingdom establish any kind of prohibition or restriction to outsourcing.

In the countries of South and Central America, there are also some restrictions on outsourcing. Thus, for example, the regulations recently adopted in Brazil prohibits outsourcing with companies owned by former workers of the user company; the Panamanian legal system prohibits outsourcing essential public services by the public administration, even though fractionation and partial decentralization of the process necessary for the provision of said services is allowed.

In Canada, there are no prohibitions nor limits to outsourcing, except in cases of transfer of undertaking and illegal posting of workers.

In the Asian legal systems analyzed (China and India), there are no general limits or prohibitions in the matter, due to the recurrent use of such forms of organization of production. However, in both cases, restrictions are established for specific economic sectors, such as the construction or financial sector, respectively.

\section{A similar divergence appears when talking about liability of main companies outsourcing their production.}


Regarding European countries analysed, on the one hand, Germany, Portugal and the United Kingdom do not generally determine any kind of liability related to outsourcing for main companies, although there are some exceptions. On the other hand, the French, the Italian and the Spanish legal regimes do foresee liabilities for user companies. However, there is not a uniform pattern regarding the scope of such liability. In France, joint liability applies, however its range shall rely on whether the outsourced works are rendered in or out de dependencies of the main company. In Italy, joint liability applies on wages, severance pay and Social Security contributions. Regarding Spain, subsidiary liability applies always for Social Security debts, but jointly liability on wages and Social Security contributions shall depend on the outsourcing of a core business activity.

The Latin American countries analyzed mainly assign to the user company some kind of responsibility regarding the fulfilment of labor obligations contracted with workers of the subcontractor: subsidiary liability in Brazil or joint liability in Peru, Uruguay and Costa Rica. The case of Panama stands out, where the user company does not have any responsibility in the fulfilment of the obligations with the workers hired by the subcontractor, with the exception of the obligations derived from the termination of the contract.

Canada foresees no obligations for the main company outsourcing its services different from health and safety obligations and liabilities when works are performed in the user company's premises.

The patterns that follow the Asian systems analyzed show notable differences. Thus, the Chinese regulation excludes, in general, the responsibility of the user company against compliance with labor obligations and differs the issue to the will of the intervening companies expressed in the respective civil contract.

4. Despite the differences of the previous questions, in the countries compared there is practical consensus regarding the collective bargaining agreement applicable to employees whose activity has been outsourced. In all the cases, the collective bargaining agreement applicable is that of the subcontractor. However, it must be said that not for all countries this fact has the same importance, since in countries such as the United Kingdom and Germany collective bargaining agreement do not have erga omnes effects.

Similarly, the predominant trend within the countries analyzed belonging to Central and South America, points to the application of the collective agreement signed by the 
contractor. In the absence of an applicable agreement at company-level, the systems of Peru, Costa Rica and Uruguay establish the application of the corresponding agreements to the respective sector or economic activity, where outsourcing takes place. Costa Rica departs from this tendency, which establishes the direct application of the collective agreement subscribed by the user company when the latter and the contractor belong to the same group of companies.

Related to Canada, the subcontractor shall apply its collective bargaining agreement. However, in cases of transferring undertakings and illegal posting of workers, the bargaining agreement of the sold business and the bargaining agreement of the main company respectively shall apply.

Regarding the Asian systems subject to comparison, there are no notable features. In China, there is no rule that regulates the matter, while in India there is a low level of coverage of the rules that arise because of the collective bargaining processes.

\section{The same consensus is found regarding the absence of an obligation to recognise the same labour conditions among subcontracted employees and employees of main companies.}

Indeed, European regulations do not oblige subcontractors to recognize their employees the same labour conditions of their clients' employees. Nevertheless, among the European countries, there are exceptions in cases of transfer of undertakings, and there might come new regulations changing the sense of these answers both in Portugal and Spain.

In Latin America, the predominant trend is the absence of a rule that guarantees equal treatment between workers of the user company and those of the contractor who provide services in the former. However, some findings allow redefining the issue. Thus, in Peru the existence of administrative decisions is reported, which, despite their restricted applicability, advocate the application of said principle; and in Brazil, equality of treatment is specifically defined in specific matters (food, first aid, remuneration for work in extra time, transportation, holidays, inter alia) when the services are carried out in the facilities of the user company.

The same tendency of lack of a principle of equal treatment in the framework of outsourcing exists in Canada, China and India.

6. In most of the countries analysed outsourcing is a legal form of production organization as long as it is not used fraudulently to avoid labor consequences. 
Generally, among European countries, there is a fraudulent outsourcing when: (i) the object of the contract is limited to the posting of workers by the subcontractor to the user company; (ii) the subcontractor is not a real enterprise, not does it have the necessary infrastructure or means of production; (iii) the user company acts as a true employer, exercising the faculties of direction, organisation and control of workers. However, two cases must be highlighted. In the United Kingdom outsourcing cannot be considered fraudulent except when both companies act under the veil of corporate activity. And Portugal allows to post indefinite workers among partner companies by written agreement for a defined period, and with the worker's consent.

The consequences of fraudulent outsourcing are also similar among European countries. In most of the cases, the consequences are joint liability of the user company and the subcontractors regarding labor and Social Security debts, the recognition of an employment relationship of the worker with the user company and, in some legal systems, administrative or criminal liability.

The irregularity or illegality of the matter in Latin America is determined, in general terms, by the absence of real cause in the business, the restriction of rights, the lack of conditions of true autonomy and independence on the part of the contractor, or the omission in compliance with the legally established requirements. Within the scope of the consequences, highlights the statement of an employment relationship between the worker and the recipient of the work performed. Some normative developments allow delimiting, with a higher level of concretion, the notion of fraud. Thus, in Peru, case law has identified, within the scope of the denaturalization assumptions of outsourcing, the performance of occupational medical examinations, or the realization of training in occupational safety and health to workers by of the user company.

In Canada (Ontario), fraudulent use of outsourcing is linked to the idea of "related employers" of the user company and the subcontractor, as a result of the identity of direction, financial control, ownership, name or trademark, market or customers, premises or workers. In these cases, case law applies the corporate veil doctrine, declaring the existence of one employer and consequently declaring joint liability of the user company and the subcontractor regarding labor debts.

In the case of China, the rules do not establish specific criteria in relation to those conducts constituting fraud, thus, the concept of subordination, specifically the form and the subject that exercises it, is relevant to resolve related disputes. Now, within the scope of the consequences, in addition to others frequently used by other systems such 
as the suspension of activities or the imposition of fines, highlights the "confiscation of profits" taxable to the responsible contractor.

\section{All European countries analysed allow the hiring of workers through} Temporary Employment Agencies. However, resorting to this activity is permitted only for temporary purposes in France, Spain and Portugal and both for permanent and temporary needs in Germany, Italy and the United Kingdom. However, in Germany is only legitimate the use of workers through Temporary Employment Agencies up to the limit of 18 consecutive months in the same company. In Italy there are quantitative limits. Concretely, permanent workers employed through an Agency can only exceed of the $20 \%$ of the permanent employees of the main company. And temporary agency workers are limited up to the $30 \%$ of the permanent employees, provided that collective bargaining agreement do not establish otherwise. Nevertheless, in France, lending of workers has recently been facilitated for young companies through new Ordonnances.

Similarly, most of the analyzed Latin American systems admit outsourcing of their activities through Temporary Employment Agencies. There are obvious coincidences in terms of the assumptions in which this is usually allowed, with increases in production or the temporary replacement of workers as the most frequent cases. However, the cases of Costa Rica and the Dominican Republic, where this phenomenon is not regulated, should be highlighted.

As to Canada, there is not time limitation whatsoever and availability contracts with Temporary Employment Agencies can be used to both temporary and permanent needs.

The legal systems of the Asian countries included in the analysis also allow the use of labor force through Temporary Employment Agencies. In India there is a certain flexibility in the matter, which enables an indefinite duration of the work contracted by this route; however, it limits the recourse to agency work when the work is aimed at carrying out tasks that are part of the company's core business or when it is used for carrying out processes of a continuous nature (perennial process). In China, this is a restricted and supplementary issue, whose appeal is justified for a specific duration of the work contracted, for the execution of tasks different from the company's core business or for the temporary replacement of workers from the user company.

8. In most of the countries under comparison there are limits or restrictions in hiring workers through Temporary Work Agencies. However, countries such as Canada (Ontario) are excluded from this general pattern. 
Among the predominant restrictions, there is a fairly relevant one that prohibits hiring agency workers to substitute workers on strike in user companies. This prohibition is embedded in all European countries analysed, with the exception of Germany that only prohibits this possibility in the construction sector. The regulations of France, Portugal and Spain and practically identical regarding this matter. The Italian regulation establishes an illegal transfer of employees, for example, when substituting a position that is suspended or affected by a reduction of working hours, and when the main company did not performed the risk assessment required by the safety and health at work Act.

In the Latin American countries analyzed, there are also limitations or prohibitions to hiring workers through Temporary Employment Agencies. The most common prohibition is the use of this form of intermediation in order to replace workers who exercise the right to strike (Brazil, Peru and Panama).

The Chinese legal system establishes two limitations or restrictions in relation to the use of Temporary Employment Agencies: the re-dispatch, whose occurrence is predicated in the event of sending workers to another company (by the user company); and the self-dispatch, which implies the establishment of temporary employment agencies by the user, in order to cover, for itself, the demand for labor power.

\section{Regarding labor and Social Security liabilities of Temporary Employment Agencies and user companies, there are no meaningful differences between the compared countries.}

Most European regulations impose liability for labor and Social Security debts to the Temporary Employment Agencies, as they are the hiring companies and employers. However, France, Germany, Portugal and Spain regulate subsidiary liability of the user company; that is, the user company's liability to fulfil labor and Social Security debts in case of insolvency of the agency. Whereas Italy establishes joint liability between the Temporary Employment Agency and the user company.

Similarly, with respect to Latin America, the study has established that the Temporary Employment Agency is, in the majority of the cases, responsible for satisfying the obligations in relation to its agency workers. However, in the region, the normative consecration of "solidarity" (Brazil, Peru, Costa Rica and Panama) is frequent, as a way to extend this responsibility to the company that benefits from the services, in cases such as the insolvency of the Agency (Brazil) or the insufficient coverage of insurances contracted to protect rights caused in favour of agency workers (Peru). 
Canada resembles in this scope to European regulations and determines subsidiary liability for labor obligations, but joint liability regarding health and safety obligations.

The cases analyzed in Asia present a similar development. Although the existing legislation in India gives the beneficiary of the contracted labor the status of main employer, the truth is that the responsibility of the user company is secondary, and is reserved for cases in which the Temporary Employment Agency does not comply with its obligations. In its turn, in the Chinese system, the Temporary Employment Agency, in addition to receiving the status of employer of the agency workers, assumes a remarkable role in the triangular relationship, which is expressed in obligations such as the resolution of disputes between the worker and the user company, or the exercise of persuasive actions, with respect to the user company, against compliance with the obligations related to health and safety at work.

\section{All European countries analyzed recognize a principle of equal treatment when hiring workers through Temporary Employment Agencies.}

In the European Union, the uniformity in the recognition of such principle is grounded on the Directive 2008/104/CE, whose article 5 establishes that conditions of posted workers shall be, at least, those applicable as if they were directly hired by the user company to fill the same position. It is interesting to highlight, however, the regulation existing in the United Kingdom, where this principle applies after 12 continuous calendar weeks in the same role during one or more assignment with the same hirer.

However, among the Latin American countries analyzed, it is possible to identify two patterns. On the one hand, South American countries mainly tend to guarantee equal treatment between workers linked to the Temporary Employment Agency and those who are directly employed by the user company, and Central American countries where anomie prevails in this area (Costa Rica, Panama and Dominican Republic).

Similarly, in the case of the Asian countries analyzed, the study also found two opposite models. On the one hand the Chinese that guarantees equal treatment and remuneration in both groups of workers; while the Hindu who, despite establishing the recognition of various benefits to agency workers, excludes them from the coverage of benefits received by workers of the user company. 


\section{2. «Top ten» conclusiones}

El Comparative Labor Law Dossier (CLLD) de este número 1/2019 de IUSLabor está enfocado en la subcontratación y las cadenas de producción e incluye artículos elaborados por un grupo de personas académicas y profesionales de reconocido prestigio en relación con este asunto.

Como es bien sabido, la externalización de la producción y de los servicios es una práctica muy extendida en la mayoría de los países y sectores económicos. Por tanto, la intención de este CLLD es analizar, desde una perspectiva comparativa, como los países están enfocando su regulación labor en relación con este tema. Concretamente, los países analizados serán Francia, Italia, Portugal, España, Reino Unido, Brasil, Costa Rica, Panamá, Perú, República Dominicana, Uruguay, Canadá, China e India.

Específicamente, los y las académicas han participado en el presente CLLD contestando a las siguientes preguntas:

1. ¿Es la descentralización productiva o subcontratación una forma lícita de organización de la producción?

2. ¿Existen límites y/o prohibiciones a la descentralización productiva?

3. La empresa principal que externaliza una parte o la totalidad de su producción, ¿mantiene alguna responsabilidad laboral o de seguridad social respecto a los trabajadores de la empresa contratista? ¿Cuáles?

4. ¿Cuál es el convenio colectivo aplicable a la empresa subcontratista durante su relación con la empresa principal?

5. ¿Existe la obligación legal de la empresa contratista de reconocer a sus trabajadores condiciones laborales aplicables a los trabajadores de la empresa principal?

6. ¿En qué supuestos existe una descentralización productiva fraudulenta o cesión ilegal de trabajadores? ¿Qué consecuencias se derivan de esta situación?

7. ¿La contratación de trabajadores mediante Empresas de Trabajo Temporal está permitida en su país? ¿En qué supuestos?

8. ¿Existen supuestos o actividades económicas en los que la contratación de trabajadores mediante Empresas de Trabajo Temporal está prohibida?

9. ¿Qué responsabilidades laborales y de Seguridad Social mantiene la Empresa de Trabajo Temporal respecto de los trabajadores cedidos a empresas usuarias? ¿Y la empresa usuaria? 
10. ¿Cómo se determinan las condiciones laborales aplicables a los trabajadores contratados mediante Empresas de Trabajo Temporal y cedidos a empresas usuarias?

A continuación, se exponen las $\mathbf{1 0}$ conclusiones más importantes en atención a estas preguntas, siguiendo por ello el mismo orden expuesto anteriormente, en relación con los artículos escritos por nuestros colaboradores internacionales.

1. En todos los países europeos, americanos y asiáticos analizados, la subcontratación es una forma legal de organización productiva. Más allá, muchos de los países analizados reconocen que este ejercicio es una expresión de la libertad de empresa protegida por sus constituciones.

Destaca la introducción reciente de legislación en el ordenamiento jurídico brasileño, donde la descentralización productiva pasó de ser considerada ilegal por la jurisprudencia, a ser aceptada por las normas legales en 2017.

2. No obstante, a pesar de que en la mayoría de los países la subcontratación es legal, algunos países recogen prohibiciones y restricciones en sus ordenamientos jurídicos a esta forma de externalización.

Sin embargo, hay que tener en cuenta que cada país tiene sus especificidades. El único país que contempla prohibiciones a la subcontratación es Italia, donde las actividades que implican riesgos en los trabajadores no pueden subcontratarse. Además, los convenios colectivos pueden introducir tanto prohibiciones como limitaciones adicionales. Otros países europeos establecen limitaciones en relación con el sector de la construcción, como son Francia y España. Alemania, por ejemplo, acaba de aprobar una ley que incorpora limitaciones a las medidas de subcontratación que impliquen la obtención, tratamiento o/y uso de datos personales. Al contrario, ni Portugal ni el Reino Unido establecen algún tipo de prohibición o restricción a la subcontratación.

En los países de Sud y Centroamérica, también existen algunas restricciones a la subcontratación. Así, por ejemplo, la normativa recientemente adoptada en Brasil prohíbe la subcontratación con empresas de propiedad de ex trabajadores de la empresa usuaria; el sistema legal panameño prohíbe la subcontratación en materia de servicios públicos esenciales por parte de la administración pública, aun cuando se admite el fraccionamiento y la descentralización parcial del proceso necesario para la prestación de dichos servicios. 
En Canadá (Ontario), no existen límites ni prohibiciones para la subcontratación, excepto en casos de transmisión de empresa y cesión ilegal de trabajadores.

En los ordenamientos jurídicos asiáticos (China e India), no se establecen límites generales o prohibiciones en la materia, debido al uso recurrente de dichas formas de organización de la producción. Sin embargo, en ambos casos, se establecen restricciones para algunos sectores económicos específicos, como el sector de la construcción o el financiero, respectivamente.

\section{La misma divergencia existe al referirse a la responsabilidad de las empresas principales que subcontratan su producción.}

En relación con los países europeos analizados, por un lado, Alemania, Portugal y el Reino Unido no establecen ningún tipo de responsabilidad para las empresas principales que externalicen su actividad, aunque existen algunas excepciones. Por otro lado, los regímenes legales de Francia, Italia y España sí que prevén responsabilidades para las empresas principales. De todas formas, no existe una perspectiva uniforme en relación con el alcance de tal responsabilidad. En Francia, está prevista la responsabilidad solidaria, aunque su alcance dependerá de si los servicios subcontratados son prestados en las instalaciones de la empresa principal o fuera de ellas. En Italia, la responsabilidad solidaria es aplicable en los salarios, la indemnización por despido y las contribuciones a la Seguridad Social. En España, la responsabilidad es subsidiaria en todo caso para las deudas con la Seguridad Social, pero la responsabilidad solidaria por los salarios y las contribuciones a la Seguridad Social dependerá de si la actividad subcontratada forma parte del core business de la principal.

Los países latinoamericanos analizados mayoritariamente asignan a la empresa usuaria algún tipo de responsabilidad respecto del cumplimiento de las obligaciones laborales contraídas con los trabajadores de la subcontratista: responsabilidad subsidiaria en Brasil o solidaridad en Perú, Uruguay y Costa Rica). Destaca el caso de Panamá, donde la empresa usuaria no tiene responsabilidad alguna en el cumplimiento de las obligaciones con los trabajadores vinculados por la subcontratación, con la excepción de las obligaciones derivadas de la extinción del contrato.

Las únicas obligaciones que prevé Canadá para la empresa principal son aquellas derivadas de la salud y la seguridad de los trabajadores cuando los servicios se presten en sus instalaciones. 
Los patrones que siguen los sistemas asiáticos analizados presentan diferencias notables. Así, la regulación china excluye, con carácter general, la responsabilidad de la empresa usuaria frente al cumplimiento de las obligaciones laborales y difiere la cuestión a la voluntad de las empresas intervinientes expresada en el respectivo contrato civil.

4. A pesar de las diferencias palpables en las cuestiones anteriores, en los países comparados existe un práctico consenso en relación con el convenio colectivo aplicable a los empleados cuya actividad ha sido externalizada. En todos los casos, es aplicable el convenio colectivo de los subcontratistas. No obstante, hay que tener en cuenta que no en todos los países este hecho tiene la misma relevancia, ya que en países como el Reino Unido o Alemania los convenios colectivos no tienen efectos erga omnes.

Similarmente, la tendencia predominante dentro de los países analizados pertenecientes a Centro y Sudamérica, apunta a la aplicación del convenio colectivo suscrito por la empresa contratista. En ausencia de convenio aplicable a ese nivel, los ordenamientos de Perú, Costa Rica y Uruguay establecen la aplicación de los convenios correspondiente al respectivo sector o actividad económica, en donde la externalización tiene lugar. De esta tendencia se aparta Costa Rica, que establece la aplicación directa del convenio colectivo suscrito por la empresa usuaria cuando esta y la empresa contratista pertenecen al mismo grupo de empresas.

Relativo a Canadá, la empresa subcontratista debe aplicar su convenio colectivo. No obstante, cuando se produce una transmisión de empresas o una cesión ilegal de trabajadores, se aplicará el convenio colectivo afectante a la actividad intercambiada y el convenio colectivo de la empresa principal.

En lo que hace referencia a los ordenamientos asiáticos sometidos a comparación, no se reportan particularidades destacables, En China no existe una norma que regule la materia, mientras que en la India se reporta un bajo nivel de cobertura de las normas que surgen como consecuencia de los procesos de negociación colectiva.

5. El mismo consenso se encuentra cuando nos referimos a la ausencia de obligación de reconocer las mismas condiciones laborales entre los trabajadores subcontratados y los trabajadores de las empresas principales.

En efecto, las legislaciones europeas no obligan a las empresas subcontratistas a reconocer a sus trabajadores las mismas condiciones que a los trabajadores de la principal. Sin embargo, existen excepciones en los países europeos en supuestos de 
sucesión de empresas y, además, pueden surgir nuevas normas al respecto que cambien el sentido de estas respuestas tanto en España como en Portugal.

En Latinoamérica, la tendencia predominante es la ausencia de norma que garantice la igualdad de trato entre los trabajadores de la empresa usuaria y aquellos trabajadores de la contratista que prestan servicios en la empresa usuaria. Sin embargo, algunos hallazgos permiten redefinir la cuestión. Así, en Perú se reporta la existencia de decisiones administrativas, que, a pesar de su aplicabilidad restringida, abogan por la aplicación de dicho principio; y en Brasil, donde se define expresamente la igualdad de trato en cuestiones específicas (alimentación, primeros auxilios, remuneración por trabajo en tiempo suplementario, transporte, vacaciones, inter alia) cuando los servicios se ejecutan en las instalaciones de la empresa usuaria.

La misma tendencia de falta de un principio de igualdad de trato en el marco de la subcontratación existe en Canadá, China e India.

6. En la mayoría de los países analizados la subcontratación es una forma legal de organización de la producción en tanto no se utilice como fórmula para eludir obligaciones laborales.

Generalmente, entre los países europeos, existe una subcontratación fraudulenta cuando: (i) el objeto de la contrata se limita a la puesta a disposición de los trabajadores de la empresa contratista a la empresa principal; (ii) la empresa contratista es una empresa ficticia, careciendo de toda infraestructura o medios productivos; o (iii) la empresa principal actúa como verdadera empresaria, al ejercer los poderes de dirección, organización y control sobre los trabajadores. No obstante, hay que destacar dos regulaciones. En el Reino Unido, la subcontratación no puede considerarse fraudulenta, excepto en los casos en que ambas empresas actúan secretamente de forma conjunta, como si de una misma empresa se tratase. Asimismo, el ordenamiento jurídico de Portugal, que permite la cesión de trabajadores entre empresas asociadas, mediante un contrato mercantil escrito, por duración determinada y con trabajadores contratados mediante un contrato indefinido y con su consentimiento.

Las consecuencias por el uso fraudulento de la subcontratación son también similares entre los países europeos; esencialmente, la responsabilidad solidaria de la empresa principal y contratista por las deudas laborales y de Seguridad Social, el reconocimiento de una relación laboral del trabajador con la empresa principal y, en ocasiones, responsabilidades administrativas o penales. 
La irregularidad o ilegalidad de la cuestión en Latinoamérica está determinada, en términos generales, por la ausencia de causa real en el negocio, la restricción de derechos, la inexistencia de condiciones de verdadera autonomía e independencia por parte de la empresa contratista, o la omisión en el cumplimiento de los requisitos legalmente establecidos. Dentro del ámbito de las consecuencias, destaca la declaración de una relación laboral entre el trabajador y el destinatario de la labor ejecutada. Algunos desarrollos normativos permiten delimitar, con mayor nivel de concreción, la noción de fraude. Así, en Perú, por vía jurisprudencial se han definido, dentro del ámbito de los supuestos de desnaturalización de la tercerización, la realización de exámenes médicos ocupacionales, o la realización de capacitaciones en materia de seguridad y salud en el trabajo a los trabajadores por parte de la empresa usuaria.

En Canadá (Ontario), el uso fraudulento de la subcontratación se vincula a supuestos de "empleadores relacionados", como consecuencia de identidad de dirección, control financiero, propiedad, denominación o logo, mercado o clientes, instalaciones o trabajadores. En estos supuestos, la jurisprudencia aplica la doctrina del levantamiento del velo, declarando la existencia de un único empleador $\mathrm{y}$, por consiguiente, imponiendo la responsabilidad solidaria por obligaciones laborales a la empresa principal y contratista.

En el caso de China, las normas no establecen criterios específicos en relación con aquellas conductas constitutivas de fraude, así, el concepto de subordinación, específicamente la forma y el sujeto que la ejerce, resulta relevante para resolver las controversias relacionadas. Ahora, dentro del ámbito de las consecuencias, además de otras frecuentemente utilizadas por otros ordenamientos como la suspensión de actividades o la imposición de multas, destaca la "confiscación de las ganancias" imponible a la empresa contratista responsable.

7. En los países europeos analizados, todas las legislaciones permiten la contratación de trabajadores a través de Empresas de Trabajo Temporal. No obstante, acudir a esta actividad solo está permitido por razones temporales en Francia, España y Portugal y por razones tanto permanentes como temporales en Alemania, Italia y el Reino Unido. No obstante, en Alemania solo es legítimo el uso de trabajadores a través de Empresas de Trabajo Temporal con el límite de 18 meses consecutivos en la misma empresa. En Italia también aplican límites cuantitativos. Concretamente, los trabajadores indefinidos contratados a través de Empresas de Trabajo Temporal solo pueden suponer el 20\% de los trabajadores indefinidos de la empresa principal. Asimismo, los trabajadores temporales provenientes de una Empresa de Trabajo Temporal están limitados al 30\% de los trabajadores indefinidos de la 
empresa principal Y, en Francia, la cesión de trabajadores ha sido recientemente facilitada para las empresas jóvenes a través de nuevas Ordonnances.

Similarmente, la mayoría de los sistemas latinoamericanos analizados admiten la externalización de sus actividades a través de Empresas de Trabajo Temporal. Existen coincidencias ostensibles en cuanto a los supuestos en los que ésta resulta habitualmente procedente, siendo los incrementos en la producción, o el reemplazo temporal de trabajadores los casos más frecuentes. Deben destacarse, sin embargo, los supuestos de Costa Rica y República Dominicana, donde este fenómeno no está regulado.

En cuanto a Canadá, no existe ningún tipo de limitación y los contratos de puesta a disposición con las Empresas de Trabajo Temporal pueden utilizarse tanto para causas temporales o permanentes.

Los ordenamientos jurídicos de los países asiáticos incluidos en el análisis también permiten el recurso a la fuerza de trabajo a través de Empresas de Trabajo Temporal. En la India existe cierta flexibilidad en la cuestión, que habilita una duración indeterminada del trabajo contratado por ésta vía; sin embargo, limita el recurso al trabajo temporal cuanto el trabajo tiene como fin la realización de tareas que forman parte del core business de la empresa o cuando se utiliza para la realización de procesos de naturaleza continua (perennial process). En la China se trata de una cuestión restringida y de carácter suplementario, cuyo recurso se justifica en una duración determinada de la labor contratada, en la ejecución de tareas diferentes del core business de la empresa o en el reemplazo temporal de trabajadores del usuario.

8. En la mayoría de los países comparados existen límites o restricciones a la contratación de empleados mediante Empresas de Trabajo Temporal. Sin embargo, países como Canadá se separan de esta premisa.

Entre las restricciones predominantes la más extendida es la prohibición de contratar a estos trabajadores para que sustituyan a trabajadores huelguistas en la empresa usuaria. Está prohibición es recogida en todos los países europeos analizados excepto Alemania, que solo prohíbe la posibilidad de acudir a una Empresa de Trabajo Temporal en el sector de la construcción. Las legislaciones de Francia, Portugal y España son prácticamente idénticas en relación con este asunto. La legislación italiana, por ejemplo, establece que existe una cesión ilegal cuando se sustituye un puesto de trabajo afectado por un expediente temporal de reducción de empleo y cuando la empresa principal no realizó la evaluación de riesgos exigida por la ley de prevención de riesgos. 
En los países latinoamericanos analizados, también existen limitaciones o prohibiciones a la contratación a través de Empresas de Trabajo Temporal. La prohibición más común es el recurso a esta forma de intermediación con el fin de reemplazar trabajadores que ejercen el derecho de huelga (Brasil, Perú y Panamá).

El ordenamiento jurídico chino se establece dos limitaciones o restricciones en relación con el uso de Empresas de Trabajo Temporal: el re-dispatch, cuya ocurrencia se predica en el evento de reenvío de trabajadores a otra compañía (por parte de la empresa usuaria); y el self-dispatch, que implica el establecimiento de agencias temporales de empleo por parte del usuario, con el fin de cubrir, para sí, la demanda de fuerza de trabajo.

9. En relación con las responsabilidades laborales y de la Seguridad Social de las Empresas de Trabajo Temporal y las empresas usuarias, no existen diferencias relevantes entre los países comparados.

La mayoría de los países europeos establecen la responsabilidad de las Empresas de Trabajo Temporal en relación con las obligaciones laborales y con la Seguridad Social de sus empleados, en la medida en que son las empresas que contratan y emplean a los trabajadores. No obstante, Francia, Alemania, Portugal y España determinan la responsabilidad subsidiaria de la empresa usuaria; esto es, la responsabilidad de la empresa usuaria para cumplir con las deudas laborales y de la Seguridad Social en caso de insolvencia de la Empresa de Trabajo Temporal. Mientras que Italia establece la responsabilidad solidaria entre la empresa cedente y la empresa usuaria.

Similarmente, con respecto a Latinoamérica, el estudio ha permitido establecer que la Empresa de Trabajo Temporal es, en el mayor de los casos analizados, la responsable de satisfacer las obligaciones emergentes en relación con los trabajadores involucrados en un proceso de cesión. Sin embargo, en la región es frecuente la consagración normativa de la "solidaridad" (Brasil, Perú, Costa Rica y Panamá), como forma de extender dicha responsabilidad a la empresa beneficiaria de los servicios, en casos como la insolvencia de la Empresa de Trabajo Temporal (Brasil) o la cobertura insuficiente de seguros contratado para amparar derechos causados en favor de aquellos trabajadores vinculados por esta vía (Perú).

Canadá se asemeja en este ámbito a las legislaciones europeas y establece una responsabilidad subsidiaria para las obligaciones laborales, pero solidaria en relación con las obligaciones sobre la salud y seguridad de los trabajadores. 
Los casos analizados en Asia presentan un desarrollo similar. Aunque la legislación existente en la India atribuye al beneficiario de la labor contratada la calidad de empleador principal, lo cierto es que la responsabilidad de aquél es secundaria, y se reserva a los casos en que la Empresa de Trabajo Temporal no cumple con las obligaciones derivadas del contrato de trabajo. A su vez, en el sistema chino, la Empresa de Trabajo Temporal, además de la asignación específica de la condición de empleador de los trabajadores enviados, asume un rol destacable en la relación triangular, que se expresa en obligaciones como la resolución de disputas entre el trabajador y la compañía usuaria, o el ejercicio de acciones persuasivas, respecto del usuario, frente al cumplimiento de las obligaciones relacionadas con la seguridad y salud en el trabajo.

\section{Todos los países europeos analizados reconocen el principio de igualdad de trato en la contratación de trabajadores mediante Empresas de Trabajo Temporal.}

Entre los países de la Unión Europea, la uniformidad en el reconocimiento de dicho principio de igualdad de trato encuentra su fundamento en la Directiva 2008/104/CE, cuyo artículo 5 establece que las condiciones de los trabajadores cedidos por Empresas de Trabajo Temporal serán, como mínimo, aquellas que les corresponderían si hubiesen sido contratados directamente por la empresa usuaria para ocupar el mismo puesto de trabajo. Es interesante, no obstante, destacar la regulación del Reino Unido, donde dicho principio se aplica después de una prestación de servicios continuada durante 12 semanas en una misma posición para una misma empresa cliente mediante uno o más contratos de puesta a disposición.

Sin embargo, entre los países de Latinoamérica analizados, es posible identificar dos patrones. De un lado, los países suramericanos mayoritariamente tendientes a garantizar la igualdad de trato entre los trabajadores vinculados a la Empresa de Trabajo Temporal y aquellos que lo están directamente por la usuaria, y los centroamericanos donde predomina la anomia en este ámbito (Costa Rica, Panamá y República Dominicana).

Similarmente, en el caso de los países asiáticos analizados, el estudio también encontró dos modelos opuestos. De un lado el chino que garantiza la igualdad de trato y remuneración en ambos grupos de trabajadores; mientras el hindú que, a pesar de establecer el reconocimiento de diversos beneficios a los trabajadores temporales, los excluye de la cobertura de beneficios causados para el personal de la empresa usuaria. 


\section{Summary table}

\subsection{Europe}

\begin{tabular}{|c|c|c|c|c|c|c|}
\hline & France & Germany & Italy & Spain & Portugal & United Kingdom \\
\hline $\begin{array}{l}\text { 1. Is outsourcing a } \\
\text { legal form of } \\
\text { production } \\
\text { organisation? }\end{array}$ & $\begin{array}{c}\text { Yes. } \\
\text { Freedom of } \\
\text { outsourcing. }\end{array}$ & $\begin{array}{c}\text { Yes. } \\
\text { Freedom of } \\
\text { outsourcing. }\end{array}$ & $\begin{array}{c}\text { Yes. } \\
\text { Freedom of } \\
\text { outsourcing. }\end{array}$ & $\begin{array}{c}\text { Yes. } \\
\text { Freedom of } \\
\text { outsourcing. }\end{array}$ & $\begin{array}{c}\text { Yes. } \\
\text { Freedom of } \\
\text { outsourcing. }\end{array}$ & Yes. \\
\hline $\begin{array}{l}\text { 2. Are there limits } \\
\text { and/or } \\
\text { prohibitions to } \\
\text { outsourcing? }\end{array}$ & $\begin{array}{c}\text { Yes. } \\
\text { Limitations: } \\
\text { construction sector. }\end{array}$ & $\begin{array}{l}\text { Yes. } \\
\text { Limitations: data } \\
\text { protection. }\end{array}$ & $\begin{array}{c}\text { Yes. } \\
\text { Prohibition: } \\
\text { risk-taking } \\
\text { activities and CBA. }\end{array}$ & $\begin{array}{c}\text { Yes. } \\
\text { Limitation: } \\
\text { construction sector. }\end{array}$ & $\begin{array}{l}\text { No. } \\
\text { No restrictions or } \\
\text { prohibition in the } \\
\text { private sector. }\end{array}$ & No. \\
\hline $\begin{array}{l}\text { 3. Does the } \\
\text { company that } \\
\text { partly or totally } \\
\text { outsources its } \\
\text { production have } \\
\text { any labor or } \\
\text { Social Security } \\
\text { responsibility in } \\
\text { relation with the } \\
\text { subcontractor's } \\
\text { workers? } \\
\text { What } \\
\text { responsibilities? }\end{array}$ & $\begin{array}{c}\text { Yes } \\
\text { Joint liability: } \\
\text { (i) independent } \\
\text { premises: wages, } \\
\text { holidays and social } \\
\text { security } \\
\text { contributions; (ii) } \\
\text { same premises: the } \\
\text { previous + } \\
\text { benefits, } \\
\text { allowances and } \\
\text { accident comp. }\end{array}$ & $\begin{array}{l}\text { No } \\
\text { Exception: transfer } \\
\text { of undertakings. }\end{array}$ & $\begin{array}{c}\text { Yes } \\
\text { Joint liability: } \\
\text { wages, severance } \\
\text { pay and Social } \\
\text { Security } \\
\text { contributions. }\end{array}$ & $\begin{array}{c}\text { Yes } \\
\text { Core business } \\
\text { activities: (i) } \\
\text { obligations prior to } \\
\text { outsourcing: } \\
\text { subsidiary liability } \\
\text { on Social Security } \\
\text { contributions (if } \\
\text { existing debts } \\
\text { certification); } \\
\text { (ii) obligations } \\
\text { during outsourcing: }\end{array}$ & $\begin{array}{l}\text { No } \\
\text { Exceptions: } \\
\text { (i) Health and } \\
\text { safety liability; } \\
\text { (ii) corporate } \\
\text { relationship; } \\
\text { (iii) independent } \\
\text { contractor 80\% } \\
\text { earnings } \\
\text { contractor. }\end{array}$ & $\begin{array}{l}\text { No. } \\
\text { Exception: transfer } \\
\text { of undertakings. }\end{array}$ \\
\hline
\end{tabular}




\begin{tabular}{|c|c|c|c|c|c|c|}
\hline & $\begin{array}{l}\text { User company: } \\
\text { duty of vigilance } \\
\text { (compliance labor } \\
\text { and social security } \\
\text { obligations). } \\
\text { Construction } \\
\text { sector: health and } \\
\text { safety liabilities. }\end{array}$ & & & $\begin{array}{c}\text { joint liability on } \\
\text { wages and social } \\
\text { security } \\
\text { contributions. } \\
\text { Other activities: } \\
\text { subsidiary liability } \\
\text { on Social Security } \\
\text { debts. }\end{array}$ & & \\
\hline $\begin{array}{l}\text { 4. What is the } \\
\text { collective } \\
\text { bargaining } \\
\text { agreement } \\
\text { applicable to the } \\
\text { contractor during } \\
\text { its relationship } \\
\text { with the main } \\
\text { company? }\end{array}$ & $\begin{array}{l}\text { CBA of the } \\
\text { subcontractor. }\end{array}$ & $\begin{array}{l}\text { CBA applicable } \\
\text { shall depend on the } \\
\text { membership of the } \\
\text { worker and } \\
\text { employer to a } \\
\text { union or } \\
\text { employer's } \\
\text { association. }\end{array}$ & $\begin{array}{l}\text { CBA of the } \\
\text { subcontractor. } \\
\text { However, peculiar } \\
\text { rules also apply. }\end{array}$ & $\begin{array}{l}\text { CBA of the } \\
\text { subcontractor. }\end{array}$ & $\begin{array}{c}\text { CBA of the } \\
\text { subcontractor. }\end{array}$ & $\begin{array}{l}\text { CBA of the } \\
\text { subcontractor. }\end{array}$ \\
\hline $\begin{array}{l}\text { 5. Is the } \\
\text { subcontractor } \\
\text { legally obliged to } \\
\text { recognize its } \\
\text { worker the same } \\
\text { labour conditions } \\
\text { applicable to the } \\
\text { workers of the } \\
\text { user company? }\end{array}$ & $\begin{array}{l}\text { No } \\
\text { Exception: transfer } \\
\text { of undertakings. }\end{array}$ & $\begin{array}{l}\text { No. } \\
\text { Exception: transfer } \\
\text { of undertaking. }\end{array}$ & $\begin{array}{l}\text { No. } \\
\text { Exception: public } \\
\text { tenders. }\end{array}$ & No. & No. & $\begin{array}{l}\text { No. } \\
\text { Exception: transfer } \\
\text { of undertakings. }\end{array}$ \\
\hline 6. In which cases & When: & When: & When user & When: & When: & No \\
\hline
\end{tabular}




\begin{tabular}{|c|c|c|c|c|c|c|}
\hline $\begin{array}{l}\text { is outsourcing } \\
\text { considered } \\
\text { fraudulent or } \\
\text { there is an illegal } \\
\text { transfer of } \\
\text { workers? } \\
\text { What are the } \\
\text { consequences? }\end{array}$ & $\begin{array}{l}\text { (i) aim of the } \\
\text { contract is posting } \\
\text { of workers; (ii) } \\
\text { contractor not real } \\
\text { company; (iii) the } \\
\text { user company acts } \\
\text { as a direct } \\
\text { employer. } \\
\text { Consequences: } \\
\text { (i) joint liability } \\
\text { labor and social } \\
\text { security } \\
\text { obligations; and } \\
\text { (ii) labor contract } \\
\text { with the user } \\
\text { company. }\end{array}$ & $\begin{array}{l}\text { (i) aim of the } \\
\text { contract is posting } \\
\text { of workers; (ii) } \\
\text { contractor not real } \\
\text { company; (iii) the } \\
\text { user company acts } \\
\text { as a direct } \\
\text { employer. } \\
\text { Consequences: } \\
\text { (i) void } \\
\text { outsourcing } \\
\text { contract; and, } \\
\text { (ii) labor contract } \\
\text { with the user } \\
\text { company. }\end{array}$ & $\begin{array}{l}\text { company acts as an } \\
\text { employer. } \\
\text { Consequences: } \\
\text { (i) labor contract } \\
\text { with the user } \\
\text { company; } \\
\text { (ii) administrative } \\
\text { liability; } \\
\text { (iii) criminal } \\
\text { liability. }\end{array}$ & $\begin{array}{l}\text { (i) aim of the } \\
\text { contract is posting } \\
\text { workers; } \\
\text { (ii) contractor not } \\
\text { real company; } \\
\text { (iii) the user } \\
\text { company acts as a } \\
\text { direct employer. } \\
\text { Consequences: } \\
\text { (i) joint liability } \\
\text { labor and Social } \\
\text { Security debts; } \\
\text { (ii) permanent } \\
\text { labor contract with } \\
\text { company of } \\
\text { worker's choice; } \\
\text { (iii) administrative } \\
\text { liability; (iv) } \\
\text { criminal liability. }\end{array}$ & $\begin{array}{l}\text { (i) aim of the } \\
\text { contract is posting } \\
\text { of workers; (ii) } \\
\text { contractor not real } \\
\text { company; (iii) the } \\
\text { user company acts } \\
\text { as a direct } \\
\text { employer. } \\
\text { Consequence: (i) } \\
\text { user company real } \\
\text { employer; (ii) } \\
\text { permanent labor } \\
\text { contract with } \\
\text { company of } \\
\text { worker's choice. } \\
\text { Legal occasional } \\
\text { employee } \\
\text { assignment: (i) } \\
\text { indefinite contract; } \\
\text { (ii) related } \\
\text { companies; (iii) } \\
\text { worker's } \\
\text { agreement; } \\
\text { (iv) limited } \\
\text { duration; (v) } \\
\text { written corporate } \\
\text { contract. }\end{array}$ & $\begin{array}{l}\text { Exception: } \\
\text { piercing of the } \\
\text { corporate veil. }\end{array}$ \\
\hline
\end{tabular}




\begin{tabular}{|c|c|c|c|c|c|c|}
\hline $\begin{array}{l}\text { 7. Is the hiring of } \\
\text { workers through } \\
\text { Temporary } \\
\text { Employment } \\
\text { Agencies allowed } \\
\text { in your country? } \\
\text { If so, in which } \\
\text { cases? }\end{array}$ & $\begin{array}{c}\text { Yes. } \\
\text { Permitted cases: } \\
\text { (i) temporary } \\
\text { substitution; } \\
\text { (ii) temporary } \\
\text { increase workload; } \\
\text { (iii) seasonal } \\
\text { employment; } \\
\text { (iv) replac. } \\
\text { entrepreneur; (v) } \\
\text { promote } \\
\text { recruitment } \\
\text { unemployed. }\end{array}$ & $\begin{array}{c}\text { Yes. } \\
\text { Both for permanent } \\
\text { and temporary } \\
\text { tasks. } \\
\text { Up to the limit of } \\
18 \text { consecutive } \\
\text { months. }\end{array}$ & $\begin{array}{l}\text { Yes. } \\
\text { Both for permanent } \\
\text { and temporary } \\
\text { tasks. } \\
\text { Quantitative limits: } \\
\text { (i) permanent } \\
\text { contract max. 20\% } \\
\text { of permanent } \\
\text { employees; } \\
\text { (ii) temporary } \\
\text { contract max. 30\% } \\
\text { of permanent } \\
\text { employees (except } \\
\text { redundant, } \\
\text { unemployed or risk } \\
\text { workers). } \\
\text { If the limits are not } \\
\text { respected, } \\
\text { Consequences: } \\
\text { (i) employment } \\
\text { contract with the } \\
\text { user firm; } \\
\text { (ii) compensation; } \\
\text { and, (iii) admin. } \\
\text { sanction. }\end{array}$ & $\begin{array}{c}\text { Yes. } \\
\text { When temporary } \\
\text { contracts are } \\
\text { permitted. }\end{array}$ & $\begin{array}{c}\text { Yes. } \\
\text { When some } \\
\text { temporary } \\
\text { contracts are } \\
\text { permitted. }\end{array}$ & $\begin{array}{c}\text { Yes. } \\
\text { Both for permanent } \\
\text { and temporary } \\
\text { tasks. }\end{array}$ \\
\hline
\end{tabular}




\begin{tabular}{|c|c|c|c|c|c|c|}
\hline $\begin{array}{c}\text { 8. Are there } \\
\text { specific cases or } \\
\text { economic } \\
\text { activities in which } \\
\text { hiring workers } \\
\text { through } \\
\text { Temporary } \\
\text { Employment } \\
\text { Agencies is limited } \\
\text { and/or } \\
\text { prohibited? }\end{array}$ & $\begin{array}{c}\text { Yes. } \\
\text { Prohibited cases: } \\
\text { (i) permanent } \\
\text { position for the } \\
\text { normal activity; (ii) } \\
6 \text { months after } \\
\text { redundancy; } \\
\text { (iii) replace } \\
\text { workers on strike. } \\
\text { Prohibited } \\
\text { activities: } \\
\text { dangerous to } \\
\text { workers health and } \\
\text { safety. }\end{array}$ & $\begin{array}{c}\text { Yes. } \\
\text { Prohibited } \\
\text { activities: } \\
\text { construction sector. }\end{array}$ & $\begin{array}{c}\text { Yes. } \\
\text { Prohibited cases: } \\
\text { (i) after } \\
\text { redundancy (6 } \\
\text { months), except } \\
\text { temporary hiring; } \\
\text { (ii) position } \\
\text { suspended or } \\
\text { reduced hours; } \\
\text { (iii) replace } \\
\text { workers on strike; } \\
\text { (absence health } \\
\text { and safety } \\
\text { assessment. }\end{array}$ & $\begin{array}{c}\text { Yes. } \\
\text { Prohibited cases: } \\
\text { (i) after dismissals } \\
\text { business reasons } \\
\text { (12 months); (ii) } \\
\text { replace workers on } \\
\text { strike; (iii) posting } \\
\text { another agency. } \\
\text { Prohibited } \\
\text { activities: } \\
\text { dangerous to } \\
\text { workers health and } \\
\text { safety. }\end{array}$ & $\begin{array}{c}\text { Yes. } \\
\text { Prohibited cases: } \\
\text { (i) after dismissals } \\
\text { business reasons } \\
\text { (12 months); } \\
\text { (ii) replace workers } \\
\text { on strike; (iii) } \\
\text { posting another } \\
\text { agency. } \\
\text { Prohibited } \\
\text { activities: } \\
\text { dangerous to } \\
\text { workers health and } \\
\text { safety. }\end{array}$ & $\begin{array}{l}\text { Prohibited cases: } \\
\text { replace workers on } \\
\text { strike. }\end{array}$ \\
\hline $\begin{array}{l}\text { 9. What labor and } \\
\text { Social Security } \\
\text { liabilities do } \\
\text { Temporary } \\
\text { Employment } \\
\text { Agencies have } \\
\text { with respect to the } \\
\text { workers hired and } \\
\text { transferred to } \\
\text { user firms? }\end{array}$ & $\begin{array}{c}\text { Temporary } \\
\text { Employment } \\
\text { Agencies: labor } \\
\text { and Social Security } \\
\text { obligations. } \\
\text { User company: } \\
\text { (i) subsidiary } \\
\text { liability labor and } \\
\text { Social Security } \\
\text { contributions; (ii) } \\
\text { health and safety } \\
\text { direct liability. }\end{array}$ & $\begin{array}{c}\text { Temporary } \\
\text { Employment } \\
\text { Agencies: labor } \\
\text { and Social Security } \\
\text { obligations. } \\
\text { User company: } \\
\text { subsidiary liability } \\
\text { Social Security } \\
\text { contributions. }\end{array}$ & $\begin{array}{c}\text { Temporary } \\
\text { Employment } \\
\text { Agencies and user } \\
\text { company: joint } \\
\text { liability labor and } \\
\text { Social Security } \\
\text { obligations. }\end{array}$ & $\begin{array}{c}\text { Temporary } \\
\text { Employment } \\
\text { Agencies: labor } \\
\text { and Social Security } \\
\text { obligations. } \\
\text { User company: } \\
\text { subsidiary liability } \\
\text { labor and Social } \\
\text { Security debts. }\end{array}$ & $\begin{array}{c}\text { Temporary } \\
\text { Employment } \\
\text { Agencies: labor } \\
\text { and Social Security } \\
\text { obligations. } \\
\text { User company: } \\
\text { (i) subsidiary } \\
\text { liability labor and } \\
\text { Social Security } \\
\text { debts; (ii) join } \\
\text { liability when } \\
\text { illegal agency. }\end{array}$ & $\begin{array}{c}\text { Temporary } \\
\text { Employment } \\
\text { Agencies: labor } \\
\text { and Social Security } \\
\text { obligations. }\end{array}$ \\
\hline
\end{tabular}




\begin{tabular}{|c|c|c|c|c|c|c|}
\hline $\begin{array}{l}\text { 10. How are the } \\
\text { labor conditions } \\
\text { applicable to } \\
\text { workers hired by } \\
\text { Temporary } \\
\text { Employment } \\
\text { Agencies and } \\
\text { transferred to } \\
\text { user companies } \\
\text { determined? }\end{array}$ & $\begin{array}{l}\text { Equal treatment } \\
\text { principle: same } \\
\text { labor conditions. }\end{array}$ & $\begin{array}{l}\text { Equal treatment } \\
\text { principle: same } \\
\text { labor conditions. } \\
\text { Exception: CBA }\end{array}$ & $\begin{array}{l}\text { Equal treatment } \\
\text { principle: same } \\
\text { labor conditions. }\end{array}$ & $\begin{array}{l}\text { Equal treatment } \\
\text { principle: same } \\
\text { labor conditions. }\end{array}$ & $\begin{array}{l}\text { Equal treatment } \\
\text { principle: same } \\
\text { labor conditions } \\
\text { (or higher } \\
\text { conditions } \\
\text { regulated by } \\
\text { agency). }\end{array}$ & $\begin{array}{l}\text { Equal treatment } \\
\text { principle: same } \\
\text { labor conditions } \\
\text { (>12 months). }\end{array}$ \\
\hline $\begin{array}{l}\text { 11. Other relevant } \\
\text { aspects and } \\
\text { personal } \\
\text { assessment of the } \\
\text { regulation } \\
\text { regarding } \\
\text { outsourcing and } \\
\text { supply chains. }\end{array}$ & $\begin{array}{c}\text { French umbrella } \\
\text { companies } \\
\text { (entrepises de } \\
\text { portage salarial): } \\
\text { innovative form } \\
\text { temporary } \\
\text { placement }\end{array}$ & - & - & - & $\begin{array}{l}\text { The regulation } \\
\text { does not observe } \\
\text { other forms of } \\
\text { productive } \\
\text { organisation. } \\
\text { New regulations } \\
\text { might come. }\end{array}$ & $\begin{array}{c}\text { Concern for } \\
\text { increase of } \\
\text { precarious work }\end{array}$ \\
\hline
\end{tabular}




\subsection{Latin America}

\begin{tabular}{|c|c|c|c|c|c|}
\hline & Brasil & Perú & Uruguay & Costa Rica & Panamá \\
\hline $\begin{array}{l}\text { 1. Is outsourcing a } \\
\text { legal form of } \\
\text { production } \\
\text { organization? }\end{array}$ & Yes. & $\begin{array}{l}\text { Yes. } \\
\text { Freedom of Enterprise. }\end{array}$ & $\begin{array}{l}\text { Yes. } \\
\text { Freedom of Enterprise. }\end{array}$ & $\begin{array}{l}\text { Yes. } \\
\text { Freedom of Enterprise. }\end{array}$ & Yes. \\
\hline $\begin{array}{l}\text { 2. Are there limits } \\
\text { and/or prohibitions to } \\
\text { outsourcing? }\end{array}$ & $\begin{array}{l}\text { Yes. } \\
\text { Prohibitions: } \\
\text { exerting subordination } \\
\text { over the workers (user } \\
\text { enterprise); } \\
\text { (ii) to perform } \\
\text { activities different than } \\
\text { those contracted; } \\
\text { (iii) incompatibility: } \\
\text { legal person owned by } \\
\text { ex workers or } \\
\text { employees (previous } \\
\text { 18 months); (iv) } \\
\text { having worked directly } \\
\text { for the user enterprise } \\
\text { (last } 18 \text { months). }\end{array}$ & $\begin{array}{l}\text { Yes. } \\
\text { Prohibition: } \\
\text { (i) restrict individual, } \\
\text { collective, and Social } \\
\text { Security rights; } \\
\text { (ii) core business } \\
\text { (case-law). }\end{array}$ & $\begin{array}{l}\text { No. } \\
\text { Prohibition: replace } \\
\text { workers covered by } \\
\text { unemployment } \\
\text { benefits (due to parcial } \\
\text { or total suspension of } \\
\text { work). } \\
\text { Collective disputes. }\end{array}$ & No. & $\begin{array}{l}\text { No. } \\
\text { Prohibition: } \\
\text { public essential } \\
\text { services. }\end{array}$ \\
\hline $\begin{array}{l}\text { 3. Does the company } \\
\text { that partly or totally } \\
\text { outsources its } \\
\text { production have any }\end{array}$ & $\begin{array}{c}\text { Yes. } \\
\text { Subsidiary liability }\end{array}$ & $\begin{array}{c}\text { Yes. } \\
\text { Joint liability: labor }\end{array}$ & $\begin{array}{l}\text { Yes. } \\
\text { Joint }\end{array}$ & Yes. & $\begin{array}{l}\text { Yes. } \\
\text { Total outsourcing: }\end{array}$ \\
\hline
\end{tabular}




\begin{tabular}{|c|c|c|c|c|c|}
\hline $\begin{array}{l}\text { labor or Social } \\
\quad \text { Security } \\
\text { responsibility in } \\
\text { relation with the } \\
\text { subcontractor's } \\
\text { workers? What } \\
\text { responsibilities? }\end{array}$ & & $\begin{array}{l}\text { and Social Security } \\
\text { debts (no collective } \\
\text { agreement or unilateral } \\
\text { obligations). } \\
\text { Continuous movement } \\
\text { of workers from } \\
\text { contractors facilities to } \\
\text { user company's is } \\
\text { required. }\end{array}$ & $\begin{array}{l}\text { liability: labour rights } \\
\text { (set by contract or } \\
\text { collective agreement), } \\
\text { social security } \\
\text { contributions, } \\
\text { occupational hazard } \\
\text { insurance fees. } \\
\text { In case of not exerting } \\
\text { the right to be informed } \\
\text { (User Enterprise) } \\
\text { Secondary liability } \\
\text { when that right is } \\
\text { exerted. } \\
\text { Labor rights }\end{array}$ & $\begin{array}{c}\text { Joint liability: } \\
\text { a. Ousourcing through } \\
\text { intermediary (company } \\
\text { which carries out a task } \\
\text { withouth possessing } \\
\text { capital) } \\
\text { b. User enterprise } \\
\text { exerts power of } \\
\text { direction } \\
\text { c. Economic interest } \\
\text { group } \\
\text { Responsibility: labor } \\
\text { and social security, } \\
\text { occupational hazard } \\
\text { insurance (rights and } \\
\text { fees). }\end{array}$ & $\begin{array}{l}\text { Rights caused up to the } \\
\text { end of the contract } \\
\text { (holidays economic } \\
\text { compensation, etc.) } \\
\text { Joint liability: } \\
\text { subcontractor } \\
\text { exclusively performs } \\
\text { tasks for the user. }\end{array}$ \\
\hline $\begin{array}{l}\text { 4. What is the } \\
\text { collective bargaining } \\
\text { agreement applicable } \\
\text { to the contractor } \\
\text { during its relationship } \\
\text { with the main } \\
\text { company? }\end{array}$ & $\begin{array}{c}\text { Outsourcing: } \\
\text { Contractor collective } \\
\text { agreement } \\
\text { Temporary } \\
\text { Employment agencies: } \\
\text { User enterprises' }\end{array}$ & $\begin{array}{l}\text { No regulation } \\
\text { Sector or contractor's } \\
\text { collective agreement } \\
\text { (constitutional basis) }\end{array}$ & $\begin{array}{l}\text { Sector or contractor's } \\
\text { collective agreement. }\end{array}$ & $\begin{array}{l}\text { Contractor's collective } \\
\text { agreement. } \\
\text { Economic group: User } \\
\text { enterprise collective } \\
\text { agreement }\end{array}$ & $\begin{array}{l}\text { Sector or contractor's } \\
\text { collective agreement }\end{array}$ \\
\hline $\begin{array}{l}5 \text {. Is the } \\
\text { subcontractor legally } \\
\text { obliged to recognize } \\
\text { its workers the same }\end{array}$ & $\begin{array}{l}\text { Yes. } \\
\text { Some of them: } \\
\text { (i) canteen; }\end{array}$ & Advances in administ. & $\begin{array}{l}\text { Yes. } \\
\text { In case of temporary } \\
\text { supply of workforce. }\end{array}$ & No legal provision. & $\begin{array}{l}\text { Minimum rights cover } \\
\text { all the workers. }\end{array}$ \\
\hline
\end{tabular}




\begin{tabular}{|c|c|c|c|c|c|}
\hline $\begin{array}{l}\text { labor conditions } \\
\text { applicable to the } \\
\text { workers of the user } \\
\text { company? }\end{array}$ & $\begin{array}{l}\text { (ii) transport services; } \\
\text { (iii) medical assistance } \\
\text { delivered at user } \\
\text { enterprise's premises; } \\
\text { (iv) training; } \\
\text { (v) health and safety at } \\
\text { the workplace. } \\
\text { In the public } \\
\text { administration: all } \\
\text { rights, if same } \\
\text { functions are } \\
\text { performed. }\end{array}$ & decisions. & & & \\
\hline $\begin{array}{l}\text { 6. In which cases is } \\
\text { outsourcing } \\
\text { considered fraudulent } \\
\text { or there is an illegal } \\
\text { transfer of workers? } \\
\text { What are the } \\
\text { consequences? }\end{array}$ & $\begin{array}{l}\text { When: } \\
\text { (i) user enterprise } \\
\text { exerts control; } \\
\text { (ii) no formal } \\
\text { requirements are } \\
\text { fulfilled; } \\
\text { (iii) length beyond } \\
\text { temporal limts. } \\
\text { Consequences } \\
\text { User enterprise } \\
\text { becomes the employer. }\end{array}$ & $\begin{array}{l}\text { When: } \\
\text { (i) aim of the contract } \\
\text { is posting of workers; } \\
\text { (ii) limit labor or Social } \\
\text { Security rights; (iii) } \\
\text { breach requirem. } \\
\text { (autonomy, resources, } \\
\text { self-managment). } \\
\text { Recently added by } \\
\text { jurisprudence. } \\
\text { When the user } \\
\text { enterprise: } \\
\text { (i) calls the } \\
\text { contractor's worker for } \\
\text { occupational } \\
\text { examination; }\end{array}$ & $\begin{array}{l}\text { When: } \\
\text { (i) subcontractors are } \\
\text { not independent of user } \\
\text { enterprises; } \\
\text { (ii) user enterprise and } \\
\text { subcontract part of the } \\
\text { same group. } \\
\text { Consequences: } \\
\text { labor contract with the } \\
\text { user company; (ii) } \\
\text { joint liability. }\end{array}$ & No regulated. & $\begin{array}{l}\text { When: } \\
\text { (i) affects rights } \\
\text { already caused; } \\
\text { (ii) made to avoid labor } \\
\text { rights (user company); } \\
\text { (iii) alter company's } \\
\text { economic structure and } \\
\text { not informed to the } \\
\text { workers; } \\
\text { (iv)transference of } \\
\text { patrimony } \\
\text { subsequently declared } \\
\text { ilegal. }\end{array}$ \\
\hline
\end{tabular}




\begin{tabular}{|c|c|c|c|c|c|}
\hline & & $\begin{array}{l}\text { (ii) provides training } \\
\text { on health and security } \\
\text { matters to the } \\
\text { contractor's worker } \\
\text { Consequences: } \\
\text { (i) labor contract with } \\
\text { the user company; } \\
\text { (ii) admin. sanctions; } \\
\text { (iii) economic } \\
\text { sanctions. }\end{array}$ & & & \\
\hline $\begin{array}{l}\text { 7. Is the hiring } \\
\text { workers through } \\
\text { Temporary } \\
\text { Employment } \\
\text { Agencies allowed in } \\
\text { your country? If so, } \\
\text { in which cases? }\end{array}$ & $\begin{array}{l}\text { Yes. } \\
\text { Permitted cases: } \\
\text { (i) temporary needs; } \\
\text { (ii) replacement } \\
\text { workers; } \\
\text { (iii) extraordinary } \\
\text { production increase. }\end{array}$ & $\begin{array}{c}\text { Yes. } \\
\text { Permitted cases: } \\
\text { (i) temporary needs; } \\
\text { (ii) replace workers. } \\
\text { Two different kinds of } \\
\text { organization } \\
\text { (enterprises and } \\
\text { cooperatives). }\end{array}$ & $\begin{array}{l}\text { It could be used in any } \\
\text { case. }\end{array}$ & $\begin{array}{c}\text { No } \\
\text { regulation. } \\
\text { Common practice. } \\
\text { Temporary or } \\
\text { permanent. }\end{array}$ & $\begin{array}{c}\text { Yes. } \\
\text { Permitted cases: } \\
\text { (i) through a } \\
\text { Specialized company; } \\
\text { (ii) } 2 \text { months (length); } \\
\text { (iii) minimum salary. } \\
\text { Joint liability: User- } \\
\text { TEA } \\
\text { Extension of } \\
\text { responsibility } \\
\text { Administ. } \\
\text { Authorization }\end{array}$ \\
\hline
\end{tabular}




\begin{tabular}{|c|c|c|c|c|c|}
\hline $\begin{array}{l}\text { 8. Are there specific } \\
\text { cases or economic } \\
\text { activities in which } \\
\text { hiring workers } \\
\text { through Temporary } \\
\text { Employment } \\
\text { Agencies is limited } \\
\text { and/or prohibited? }\end{array}$ & $\begin{array}{c}\text { Yes. } \\
\text { Prohibited cases: } \\
\text { (i) replace workers on } \\
\text { strike; } \\
\text { (ii) foreigner workers } \\
\text { with provisional visa. }\end{array}$ & $\begin{array}{l}\text { Yes. } \\
\text { Prohibited case: } \\
\text { replace workers on } \\
\text { strike }\end{array}$ & $\begin{array}{c}\text { Yes. } \\
\text { Prohibited cases: } \\
\text { replacing workers } \\
\text { covered by } \\
\text { unemployment benefits } \\
\text { (due to parcial or total } \\
\text { suspension of work). }\end{array}$ & $\begin{array}{c}\text { No regulation. } \\
\text { Common practice. }\end{array}$ & $\begin{array}{c}\text { Yes. } \\
\text { Prohibited cases: (i) } \\
\text { Delivery of workers to } \\
\text { a foreigner country; (ii) } \\
\text { Public employment; } \\
\text { (iii) Replace workers } \\
\text { on strike. }\end{array}$ \\
\hline $\begin{array}{l}\text { 9. What labor and } \\
\text { Social Security } \\
\text { liabilities does the } \\
\text { Temporary } \\
\text { Employment } \\
\text { Agencies have with } \\
\text { respect to the workers } \\
\text { hired and transfer to } \\
\text { user firms? And the } \\
\text { user firm? }\end{array}$ & $\begin{array}{l}\text { Subsidiary liability } \\
\text { (user enterprise is } \\
\text { responsible of rights } \\
\text { not afforded by the } \\
\text { TEA. } \\
\text { Joint liability: } \\
\text { insolvency of TEA. }\end{array}$ & $\begin{array}{l}\text { Temporary Work } \\
\text { Agencies: } \\
\text { liability salary, labor } \\
\text { conditions, health and } \\
\text { safety workplace. } \\
\text { Joint liability: on } \\
\text { labour rights not } \\
\text { covered by insurance. }\end{array}$ & $\begin{array}{l}\text { Temporary Work } \\
\text { Agencies: as a true } \\
\text { employer holds all } \\
\text { responsibility. }\end{array}$ & $\begin{array}{l}\text { Contractor and User } \\
\text { company. } \\
\text { joint liability on labor } \\
\text { and social security } \\
\text { rights agency acts. }\end{array}$ & $\begin{array}{l}\text { Temporary Work } \\
\text { Agencies: exclusive } \\
\text { responsibility on social } \\
\text { security matters. } \\
\text { Labor rights: joint } \\
\text { liability TEA and user. }\end{array}$ \\
\hline $\begin{array}{c}\text { 10. How are the labor } \\
\text { conditions applicable } \\
\text { to workers hired by } \\
\text { Temporary } \\
\text { Employment } \\
\text { Agencies and } \\
\text { transferred to user } \\
\text { companies }\end{array}$ & $\begin{array}{l}\text { Equal treatment, } \\
\text { specific labor } \\
\text { conditions: } \\
\text { (i) wages; } \\
\text { (ii) work day limits } \\
\text { (extra time); } \\
\text { (iii) holidays; } \\
\text { (iv) weekly rest; }\end{array}$ & $\begin{array}{c}\text { Equal treatment } \\
\text { principle: same labor } \\
\text { conditions. }\end{array}$ & $\begin{array}{l}\text { Equal treatment } \\
\text { principle: same labor } \\
\text { benefits set for the } \\
\text { specific category of } \\
\text { work. }\end{array}$ & No regulation. & $\begin{array}{l}\text { No regulation } \\
\text { Unequal treatment as a } \\
\text { matter of fact. }\end{array}$ \\
\hline
\end{tabular}




\begin{tabular}{|c|c|c|c|c|c|}
\hline determined? & $\begin{array}{l}\text { (v) night work } \\
\text { compensation; } \\
\text { (vii) unfair dismissal, } \\
\text { and extinction } \\
\text { compensation; } \\
\text { (viii) work accident } \\
\text { insurance; } \\
\text { (ix) Social Security } \\
\text { rights. }\end{array}$ & & & & \\
\hline $\begin{array}{l}\text { 11. Other relevant } \\
\text { aspects and personal } \\
\text { assessment of the } \\
\text { regulation regarding } \\
\text { outsourcing and } \\
\text { supply chains }\end{array}$ & - & $\begin{array}{l}\text { Right to information. } \\
\text { Workers subject to } \\
\text { outsourcing are entitled } \\
\text { to know: i) the user } \\
\text { enterprise identity; ii) } \\
\text { The activities agreed in } \\
\text { the contract; iii) The } \\
\text { place where activities } \\
\text { are carried out } \\
\text { User company: inform } \\
\text { union contractor's } \\
\text { identity and movement } \\
\text { of workers. }\end{array}$ & - & - & - \\
\hline
\end{tabular}




\subsection{North America}

\begin{tabular}{|c|c|}
\hline & Canada \\
\hline $\begin{array}{c}\text { 1. Is outsourcing a legal form of production } \\
\text { organisation? }\end{array}$ & $\begin{array}{l}\text { Yes. } \\
\text { Freedom of outsourcing. }\end{array}$ \\
\hline $\begin{array}{l}\text { 2. Are there limits and/or prohibitions to } \\
\text { outsourcing? }\end{array}$ & $\begin{array}{l}\text { No. } \\
\text { Exceptions: (i) transfer of undertakings; (ii) illegal posting of workers. }\end{array}$ \\
\hline $\begin{array}{l}\text { 3. Does the company that partly or totally } \\
\text { outsources its production have any labor or Social } \\
\text { Security responsibility in relation with the } \\
\text { subcontractor's workers? } \\
\text { What responsibilities? }\end{array}$ & $\begin{array}{l}\text { No. } \\
\text { Exception: health and safety obligations and liabilities when work is performed in user company's premises. }\end{array}$ \\
\hline $\begin{array}{l}\text { 4. What is the collective bargaining agreement } \\
\text { applicable to the contractor during its relationship } \\
\text { with the main company? }\end{array}$ & $\begin{array}{l}\text { CBA of the subcontractor. } \\
\text { Exceptions: (i) transfer of undertaking, CBA of the sold business; (ii) illegal posting of workers, CBA of the main } \\
\text { company. }\end{array}$ \\
\hline $\begin{array}{l}\text { 5. Is the subcontractor legally obliged to recognize } \\
\text { its worker the same labour conditions applicable to } \\
\text { the workers of the user company? }\end{array}$ & No. \\
\hline $\begin{array}{l}\text { 6. In which cases is outsourcing considered } \\
\text { fraudulent or there is an illegal transfer of } \\
\text { workers? What are the consequences? }\end{array}$ & $\begin{array}{l}\text { When user company and subcontractor are "related employers": common management, financial control, } \\
\text { ownership, trade name or logo, market customers, premises or workers. } \\
\text { Consequences: (i) one employer; (ii) joint liability for employment obligations. }\end{array}$ \\
\hline
\end{tabular}




\begin{tabular}{|c|c|}
\hline $\begin{array}{l}\text { 7. Is the hiring of workers through Temporary } \\
\text { Employment Agencies allowed in your country? } \\
\text { If so, in which cases? }\end{array}$ & $\begin{array}{l}\text { Yes. } \\
\text { No time limitation: temporary or permanent. }\end{array}$ \\
\hline $\begin{array}{l}\text { 8. Are there specific cases or economic activities in } \\
\text { which hiring workers through Temporary } \\
\text { Employment Agencies is limited and/or prohibited? }\end{array}$ & $\begin{array}{l}\text { No. } \\
\text { Only restrictions on the relationship between worker and agency: (i) prohibition charging fees; (ii) allow direct } \\
\text { employment user firm (except "buyout" fees } 6 \text { months); (iii) allow reference user firm; (iv) written information. }\end{array}$ \\
\hline $\begin{array}{l}\text { 9. What labor and Social Security liabilities do } \\
\text { Temporary Employment Agencies have with } \\
\text { respect to the workers hired and transferred to user } \\
\text { firms? }\end{array}$ & $\begin{array}{l}\text { Temporal Employment Agencies: labor and Social Security obligations. } \\
\text { User company: (i) subsidiary liability labor obligations; (ii) joint liability health and safety obligations. }\end{array}$ \\
\hline $\begin{array}{c}\text { 10. How are the labor conditions applicable to } \\
\text { workers hired by Temporary Employment } \\
\text { Agencies and transferred to user companies } \\
\text { determined? }\end{array}$ & Minimum legal labor standards. + special rules in certain labor conditions. \\
\hline $\begin{array}{l}\text { 11. Other relevant aspects and personal assessment } \\
\text { of the regulation regarding outsourcing and supply } \\
\text { chains. }\end{array}$ & Jurisdiction of Ontario. \\
\hline
\end{tabular}


3.4. Asia

\begin{tabular}{|c|c|c|}
\hline & China & India \\
\hline $\begin{array}{c}\text { 1. Is outsourcing a legal form of production } \\
\text { organization? }\end{array}$ & Yes. & $\begin{array}{l}\text { Yes. } \\
\text { Different legal sources (National telecom policy, } \\
\text { contract act, companies act, copyright act, } \\
\text { patents act, foreigner exchange management act, } \\
\text { labour legislation, etc.). }\end{array}$ \\
\hline $\begin{array}{l}\text { 2. Are there limits and/or prohibitions to } \\
\text { outsourcing? }\end{array}$ & $\begin{array}{l}\text { Yes. } \\
\text { Prohibitions: } \\
\text { Contract letting party- construction sector: } \\
\text { (i) outsourcing the project to one individual; } \\
\text { (ii) outsourcing to an agent without qualifications; } \\
\text { (iii) the outsourcing fails to follow legal } \\
\text { procedures; } \\
\text { (iv) setting of unreasonable conditions for } \\
\text { tendering or bidding, in order to exclude eventual } \\
\text { participants of the process; } \\
\text { (v) division of a project into parts, and the } \\
\text { outsourcing to general undertakings, or specialized } \\
\text { contractor undertakings. } \\
\text { Contract undertaking: } \\
\text { (i) subcontracting to an individual; } \\
\text { (ii) subcontracting to an agent without } \\
\text { qualifications; } \\
\text { (iii) subcontracting of the main structure of the }\end{array}$ & $\begin{array}{l}\text { Limits: } \\
\text { Previous registration } \\
\text { Prohibition: core services of certain sectors in the } \\
\text { insurance, banking }\end{array}$ \\
\hline
\end{tabular}




\begin{tabular}{|c|c|c|}
\hline & $\begin{array}{l}\text { construction project (except steel works); } \\
\text { (iv) subcontracting of non-labor operation; } \\
\text { (v) subcontracting of labor services to other } \\
\text { parties; } \\
\text { (vi) calculation of fees for major construction } \\
\text { materials, mixing of materials, and large-medium } \\
\text { sized construction machinery and equipment. }\end{array}$ & \\
\hline $\begin{array}{l}\text { 3. Does the company that partly or totally } \\
\text { outsources its production have any labor or } \\
\text { Social Security responsibility in relation } \\
\text { with the subcontractor's workers? What } \\
\text { responsibilities? }\end{array}$ & $\begin{array}{c}\text { No (general rule). } \\
\text { Exceptions: } \\
\text { (i) stipulation in the civil contract (user- } \\
\text { subcontractor); } \\
\text { (ii) outsourcing to contractor which recruits } \\
\text { against provisions of labor law and cause damages } \\
\text { to employees; } \\
\text { (iii) outsourcing to contractor without legal } \\
\text { employer qualification } \\
\text { Subsidiary liability. }\end{array}$ & $\begin{array}{l}\text { No obligation of contribution to social security } \\
\text { (pension) for nationals (when payment > wage } \\
\text { ceiling). }\end{array}$ \\
\hline $\begin{array}{l}\text { 4. What is the collective bargaining } \\
\text { agreement applicable to the contractor } \\
\text { during its relationship with the main } \\
\text { company? }\end{array}$ & $\begin{array}{l}\text { No regulation. } \\
\text { Individual labor contract is subject to contractor } \\
\text { collective agreement. }\end{array}$ & $\begin{array}{c}\text { Low coverage of collective agreements to } \\
\text { workforce. }\end{array}$ \\
\hline $\begin{array}{l}\text { 5. Is the subcontractor legally obliged to } \\
\text { recognize its workers the same labor } \\
\text { conditions applicable to the workers of the } \\
\text { user company? }\end{array}$ & $\begin{array}{l}\text { Equal pay for work of equal value is applicable to } \\
\text { workers in the same company. }\end{array}$ & $\begin{array}{l}\text { Legal vacuum. } \\
\text { Factual differences between contractor's and } \\
\text { user's workers. }\end{array}$ \\
\hline
\end{tabular}




\begin{tabular}{|c|c|c|}
\hline & & Proposed amendment to level off differences. \\
\hline $\begin{array}{l}\text { 6. In which cases is outsourcing considered } \\
\text { fraudulent or there is an illegal transfer of } \\
\text { workers? What are the consequences? }\end{array}$ & $\begin{array}{l}\text { When: } \\
\text { (i) using outsourced workers in the form of labor } \\
\text { dispatch; } \\
\text { (ii) evaluation of Management of core interests; } \\
\text { (iii) calculating method of outsourcing fee } \\
\text { Consequences: } \\
\text { (i) subcontractor is considered to be the employer, } \\
\text { and the company outsourcing as the user } \\
\text { undertaking of a labor dispatch; } \\
\text { (ii) joint liability; } \\
\text { (iii) labor administrative sanctions. }\end{array}$ & $\begin{array}{l}\text { Outsourcing of core services of certain sectors } \\
\text { (finances, insurance). } \\
\text { No provisions on transfer of employees. }\end{array}$ \\
\hline $\begin{array}{l}\text { 7. Is the hiring workers through } \\
\text { Temporary Employment Agencies allowed } \\
\text { in your country? If so, in which cases? }\end{array}$ & $\begin{array}{l}\text { Yes. } \\
\text { Permitted cases: } \\
\text { (i) temporary positions (no longer than } 6 \text { months); } \\
\text { (ii) auxiliary positions (different than core } \\
\text { activities); } \\
\text { (iii) substitutive positions (to replace temporary } \\
\text { absences). } \\
\text { Dispatch cannot exceed } 10 \% \text { of the total } \\
\text { employment. }\end{array}$ & $\begin{array}{l}\text { Yes. } \\
\text { Frequently used. Skilled workers. }\end{array}$ \\
\hline
\end{tabular}




\begin{tabular}{|c|c|c|}
\hline $\begin{array}{l}\text { 8. Are there specific cases or economic } \\
\text { activities in which hiring workers through } \\
\text { Temporary Employment Agencies is } \\
\text { limited and/or prohibited? }\end{array}$ & $\begin{array}{l}\text { Yes. } \\
\text { Prohibitions: } \\
\text { (i) re-dispatch, (delivery of dispatched workers to } \\
\text { another user undertaking); } \\
\text { (ii) self-dispatch (establishment of agencies to } \\
\text { dispatch workers to the same company). }\end{array}$ & $\begin{array}{l}\text { Yes. } \\
\text { Prohibited case: if the work is core to the } \\
\text { industry or if is perennial process. }\end{array}$ \\
\hline $\begin{array}{l}\text { 9. What labor and Social Security liabilities } \\
\text { does the Temporary Employment Agencies } \\
\text { have with respect to the workers hired and } \\
\text { transfer to user firms? And the user firm? }\end{array}$ & $\begin{array}{l}\text { Temporary Work Agencies: as a true employer } \\
\text { holds all the responsibilities. } \\
\text { Obligations: } \\
\text { than two years; } \\
\text { (i) extinction fix-term contract if is longer } \\
\text { (ii) informing worker over relevant facts of the } \\
\text { labor contract, training system, including } \\
\text { safety and education training; } \\
\text { (iii) salary, perks, and social security contributions; } \\
\text { (iv) payment of salaries when not available work; } \\
\text { (v) providing labor protection, working safety and } \\
\text { health conditions; } \\
\text { (vi) proof for rescission or termination of the } \\
\text { contract; } \\
\text { assistance in disputes between the } \\
\text { (vii) dispatched worker and user undertaking. } \\
\text { User company: } \\
\text { (i) providing the necessary working } \\
\text { conditions and occupational protection; }\end{array}$ & $\begin{array}{l}\text { Temporary Work Agencies: } \\
\text { Payment of salary and benefits. } \\
\text { User enterprise is considered the principal } \\
\text { employer. It must pay salaries and benefits in } \\
\text { case of no pay by TEA. Statutory right to recover } \\
\text { the amount paid. }\end{array}$ \\
\hline
\end{tabular}




\begin{tabular}{|c|c|c|}
\hline & $\begin{array}{c}\text { (ii) informing dispatched workers of the job } \\
\text { requirements and labor remuneration; } \\
\text { (iii) offering overtime pay, performance bonuses, } \\
\text { and welfare benefits; } \\
\text { (iv) providing with necessary training of the } \\
\text { working position; } \\
\text { (v) applying regular wage adjustment mechanisms } \\
\text { in case of continued employment. }\end{array}$ & \\
\hline $\begin{array}{l}\text { 10. How are the labor conditions applicable } \\
\text { to workers hired by Temporary } \\
\text { Employment Agencies and transferred to } \\
\text { user companies determined? }\end{array}$ & $\begin{array}{l}\text { By law. } \\
\text { Equal treatment principle: same labor conditions }\end{array}$ & By law. \\
\hline $\begin{array}{l}\text { 11. Other relevant aspects and personal } \\
\text { assessment of the regulation regarding } \\
\text { outsourcing and supply chains. }\end{array}$ & & $\begin{array}{l}\text { Right to information. } \\
\text { Workers subject to outsourcing are entitled to } \\
\text { know: } \\
\text { (i) the user enterprise identity; } \\
\text { ii) the activities agreed in the contract; } \\
\text { (iii) the place where activities are carried out. } \\
\text { The user enterprise must inform the union of the } \\
\text { contractor's identity and movement of workers. }\end{array}$ \\
\hline
\end{tabular}

